\title{
Universiteit
}

Leiden

The Netherlands

\section{Aggressive fluid hydration plus non-steroidal anti-inflammatory drugs versus non-steroidal anti-inflammatory drugs alone for post- endoscopic retrograde cholangiopancreatography pancreatitis (FLUYT) a multicentre, open-label, randomised, controlled trial} Weiland, C.J.S.; Smeets, X.J.N.M.; Kievit, W.; Verdonk, R.C.; Poen, A.C.; Bhalla, A.; ... ; Dutch Pancreatitis Study G

\section{Citation}

Weiland, C. J. S., Smeets, X. J. N. M., Kievit, W., Verdonk, R. C., Poen, A. C., Bhalla, A., ... Geenen, E. J. M. van. (2021). Aggressive fluid hydration plus non-steroidal antiinflammatory drugs versus non-steroidal anti-inflammatory drugs alone for post-endoscopic retrograde cholangiopancreatography pancreatitis (FLUYT): a multicentre, open-label, randomised, controlled trial. The Lancet Gastroenterology \& Hepatology, 6(5), 350-358. doi:10.1016/S2468-1253(21)00057-1

Version: Publisher's Version

License: $\quad$ Creative Commons CC BY 4.0 license

Downloaded from: https://hdl.handle.net/1887/3196208

Note: To cite this publication please use the final published version (if applicable). 


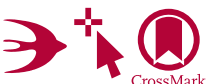 Aggressive fluid hydration plus non-steroidal anti- inflammatory drugs versus non-steroidal anti-inflammatory drugs alone for post-endoscopic retrograde cholangiopancreatography pancreatitis (FLUYT): a multicentre, open-label, randomised, controlled trial
}

\author{
Christina J Sperna Weiland*, Xavier J N M Smeets*, Wietske Kievit, Robert C Verdonk, Alexander C Poen, Abha Bhalla, Niels G Venneman, \\ Ben J M Witteman, David W da Costa, Brechje C van Eijck, Matthijs P Schwartz, Tessa E H Römkens, Jan Maarten Vrolijk, Muhammed Hadithi, \\ Annet M C J Voorburg, Lubbertus C Baak, Willem J Thijs, Roy L van Wanrooij, Adriaan C IT L Tan, Tom CJ Seerden, Yolande C A Keulemans, \\ Thomas R de Wijkerslooth, Wim van de Vrie, Peter van der Schaar, Sven M van Dijk, Nora D L Hallensleben, Ruud L Sperna Weiland, \\ Hester C Timmerhuis, Devica S Umans, Jeanin Evan Hooft, Harry van Goor, Hjalmar C van Santvoort, Marc G Besselink, MarcoJ Bruno, \\ Paul Fockens, Joost P H Drenth, Erwin J M van Geenen, on behalf of the Dutch Pancreatitis Study Group
}

\section{Summary}

Lancet Gastroenterol Hepatol 2021; 6: 350-58

Published Online

March 16, 2021

https://doi.org/10.1016/ S2468-1253(21)00057-1

See Comment page 336

${ }^{*}$ Contributed equally

Department of Gastroenterology and Hepatology

(C) Sperna Weiland MD,

XJ N M Smeets MD,

ProfJPH Drenth MD,

E) M van Geenen MD),

Department for Health Evidence (W Kievit MD), and Department of Surgery (Prof H van Goor MD),

Radboud University Medical Center, Nijmegen, Netherlands; Department of Research and Development

(C) Sperna Weiland, X J N M Smeets, S M van Dijk MD, N D L Hallensleben MD, HCTimmerhuis MD, D S Umans BSc), Department of Gastroenterology and Hepatology (RCVerdonk MD, $P$ van der Schaar MD),

Department of Radiology

(DW da Costa MD), and

Department of Surgery

(Prof H C van Santvoort MD), St Antonius Hospital,

Nieuwegein, Netherlands; Department of

Gastroenterology and Hepatology, Isala Clinics, Zwolle, Netherlands (A C Poen MD); Department of Gastroenterology and Hepatology, Hagaziekenhuis,

The Hague, Netherlands (A Bhalla MD); Department of Gastroenterology and Hepatology, Medisch Spectrum

Background Pancreatitis is the most common complication of endoscopic retrograde cholangiopancreatography (ERCP). Prophylactic rectal administration of non-steroidal anti-inflammatory drugs (NSAIDs) is considered as standard of care to reduce the risk of post-ERCP pancreatitis. It has been suggested that aggressive hydration might further reduce this risk. Guidelines already recommend aggressive hydration in patients who are unable to receive rectal NSAIDs, although it is laborious and time consuming. We aimed to evaluate the added value of aggressive hydration in patients receiving prophylactic rectal NSAIDs.

Methods FLUYT, a multicentre, open-label, randomised, controlled trial done across 22 Dutch hospitals, included patients aged between 18 and 85 years with moderate to high risk of post-ERCP pancreatitis. Patients were randomly assigned (1:1) by a web-based module with varying block sizes to a combination of aggressive hydration and rectal NSAIDs (100 mg diclofenac or indomethacin; aggressive hydration group) or rectal NSAIDs (100 $\mathrm{mg}$ diclofenac or indomethacin) alone (control group). Randomisation was stratified according to treatment centre. Aggressive hydration comprised $20 \mathrm{~mL} / \mathrm{kg}$ intravenous Ringer's lactate solution within $60 \mathrm{~min}$ from the start of ERCP, followed by $3 \mathrm{~mL} / \mathrm{kg}$ per $h$ for $8 \mathrm{~h}$. The control group received normal intravenous saline with a maximum of $1.5 \mathrm{~mL} / \mathrm{kg} \mathrm{per} \mathrm{h}$ and $3 \mathrm{~L}$ per $24 \mathrm{~h}$. The primary endpoint was post-ERCP pancreatitis and was analysed on a modified intention-to-treat basis (including all patients who underwent randomisation and an ERCP and for whom data regarding the primary outcome were available). The trial is registered with the ISRCTN registry, ISRCTN13659155.

Findings Between June 5, 2015, and June 6, 2019, 826 patients were randomly assigned, of whom 388 in the aggressive hydration group and 425 in the control group were included in the modified intention-to-treat analysis. Post-ERCP pancreatitis occurred in $30(8 \%)$ patients in the aggressive hydration group and in $39(9 \%)$ patients in the control group (relative risk $0 \cdot 84,95 \% \mathrm{CI} 0 \cdot 53-1 \cdot 33, \mathrm{p}=0 \cdot 53$ ). There were no differences in serious adverse events, including hydration-related complications (relative risk 0.99, 95\% CI 0.59-1.64; $\mathrm{p}=1 \cdot 00)$, ERCP-related complications $(0 \cdot 90$, $0 \cdot 62-1 \cdot 31 ; \mathrm{p}=0 \cdot 62)$, intensive care unit admission $(0 \cdot 37,0 \cdot 07-1 \cdot 80 ; \mathrm{p}=0 \cdot 22)$, and 30-day mortality $(0 \cdot 95,0 \cdot 50-1 \cdot 83$ $\mathrm{p}=1 \cdot 00)$.

Interpretation Aggressive periprocedural hydration did not reduce the incidence of post-ERCP pancreatitis in patients with moderate to high risk of developing this complication who routinely received prophylactic rectal NSAIDs Therefore, the burden of laborious and time-consuming aggressive periprocedural hydration to further reduce the risk of post-ERCP pancreatitis is not justified.

Funding Netherlands Organisation for Health Research and Development and Radboud University Medical Center.

Copyright (C) 2021 Elsevier Ltd. All rights reserved.

Introduction

In the USA alone, 169510 endoscopic retrograde cholangiopancreatographies (ERCPs) are done annually. Pancreatitis is the most common complication of ERCP, with an incidence of up to $14.7 \%$ in patients at high risk. Post-ERCP pancreatitis can progress to moderate or severe pancreatitis in $4.7 \%$ of patients and is associated with an overall mortality rate of $0 \cdot 7 \% .^{2,3}$ 
Research in context

\section{Evidence before this study}

Pancreatitis is the most common complication of endoscopic retrograde cholangiopancreatography (ERCP). Despite the use of prophylactic rectal non-steroidal anti-inflammatory drugs (NSAIDs), its prevalence remains substantial. Evidence has emerged that aggressive periprocedural hydration using Ringer's lactate solution is also effective in reducing post-ERCP pancreatitis. Therefore, we did a systemic review before trial commencement using PubMed and Embase to search for research articles published in English up to Feb 17, 2016, with the following search terms: ("cholangiopancreatography, endoscopic retrograde", "ERCP") and ("fluid therap*", "fluid administrat", "fluid volume", "intravenous infusion", "rehydrate", or "hydrat*"). Six studies with a total of 1102 patients fulfilled the inclusion criteria: three randomised controlled trials and three retrospective studies. On the basis of this systematic review, there was evidence to suggest that periprocedural hydration affords protection against post-ERCP pancreatitis. However, the included studies did not use prophylactic rectal NSAIDs, which are now seen as the standard of care. Furthermore, the pooled sample size of the included studies was too small to detect differences in post-ERCP pancreatitis. Finally, the included studies had considerable shortcomings in methodology, reporting confounders, and various endpoint definitions.

\section{Added value of this study}

In this multicentre, randomised, controlled trial, we found that that the combination therapy with aggressive periprocedural hydration with Ringer's lactate solution and rectal NSAIDs was not superior to rectal NSAIDs alone in reducing the incidence of post-ERCP pancreatitis.

Implications of all the available evidence

In patients undergoing ERCP who already receive prophylactic rectal NSAIDs, the burden of laborious and time-consuming aggressive periprocedural hydration to further reduce the risk of post-ERCP pancreatitis is not justified. Aggressive periprocedural hydration can potentially be used in patients with contraindications for rectal NSAIDs.

Effective strategies to reduce the incidence of post-ERCP pancreatitis are periprocedural rectal non-steroidal antiinflammatory drugs (NSAIDs) and pancreatic duct stent placement. ${ }^{4-6}$ Rectal NSAIDs are widely regarded as the standard preventive therapy because of ease of use and negligible costs. ${ }^{7-9}$ There is insufficient evidence, however, that a combination of rectal NSAIDs and pancreatic duct stenting is superior to either technique alone., ${ }^{7,10-12}$ Despite these routine preventive measures, pancreatitis remains the most common complication of ERCP. ${ }^{5}$ Evidence has emerged that aggressive periprocedural hydration with Ringer's lactate solution is also effective and safe in reducing post-ERCP pancreatitis. ${ }^{13-19}$ Therefore, American endoscopy treatment guidelines suggest aggressive periprocedural hydration with Ringer's lactate solution when feasible to decrease the risk of post-ERCP pancreatitis, and European guidelines advise aggressive periprocedural hydration in patients with a contraindication for rectal NSAIDs. ${ }^{8}$

A survey among American endoscopists involved in advanced endoscopy fellowships reported that $83 \%$ of the responders use intravenous fluids to prevent post-ERCP pancreatitis. ${ }^{20}$ The strategy is rooted in the theory that early derangements of pancreatic microcirculatory perfusion correlate with acute pancreatitis severity. ${ }^{21}$ However, the question remains whether periprocedural hydration offers protection in patients who are already receiving rectal NSAIDs. A synergistic effect of hydration and rectal NSAIDs cannot be excluded because hydration aims to preserve pancreatic microcirculation while NSAIDs suppress the inflammatory response. ${ }^{21-24}$ Studies of aggressive periprocedural hydration as an addition to rectal NSAIDs have not provided robust conclusions..$^{25,26}$
Since aggressive hydration is laborious, time consuming, and often necessitates a prolonged hospital stay, a randomised study is needed to determine the effectiveness of such a strategy.

We aimed to compare aggressive periprocedural hydration with Ringer's lactate solution combined with rectal NSAIDs versus rectal NSAIDs alone in patients undergoing ERCP with a moderate to high risk of postERCP pancreatitis.

\section{Methods}

\section{Study design and participants}

FLUYT, a multicentre, open-label, randomised, controlled trial, was coordinated by the Dutch Pancreatitis Study Group. Patients were enrolled in four university medical centres and 18 large teaching hospitals in the Netherlands. The design of the trial has been published previously. ${ }^{27}$

Patients aged between 18 and 85 years were eligible for inclusion if they needed ERCP and they had a moderate to high risk of post-ERCP pancreatitis. We selected patients at moderate to high risk of post-ERCP pancreatitis by excluding patients with a low risk of post-ERCP pancreatitis, for which they had to fulfil at least one of the following criteria: chronic calcific pancreatitis (according to M-ANNHEIM criteria ${ }^{28}$ ), previous sphincterotomy, pancreatic head mass, or routine biliary stent exchange., Additional exclusion criteria were active pancreatitis before ERCP and contraindications to aggressive hydration (eg, cardiac, pulmonic, or liver insufficiency, pre-existent pitting oedema, hyponatraemia, or hypernatraemia) or rectal NSAIDs (eg, renal insufficiency, allergy, active gastrointestinal bleeding, ulcer disease, and NSAID use for other indications [other than
Twente, Enschede, Netherlands

(N GVenneman MD);

Department of

Gastroenterology and Hepatology, Gelderse Vallei Hospital, Ede, Netherlands (Prof B J M Witteman MD); Department of Gastroenterology and Hepatology, Spaarne Gasthuis, Hoofddorp, Netherlands (B C van Eijck MD); Department of Gastroenterology and Hepatology, Meander Medica Centre, Amersfoort, Netherlands (M P Schwartz MD); Department of Gastroenterology and Hepatology, Jeroen Bosch Hospital, Den Bosch, Netherlands

(T E H Römkens MD); Department of Gastroenterology and Hepatology, Rijnstate Hospital, Arnhem, Netherlands (J M Vrolijk MD); Department of Gastroenterology and Hepatology, Maasstad Hospital, Rotterdam Netherlands (M Hadithi MD); Department of Gastroenterology and Hepatology, Diakonessenhuis, Utrecht, Netherlands (A M C J Voorburg MD); Department of Gastroenterology and Hepatology, Onze Lieve Vrouwe Gasthuis, Amsterdam Netherlands (LC Baak MD); Department of Gastroenterology and Hepatology, Martini Hospital, Groningen, Netherlands (WJThijs MD); Department of Gastroenterology and Hepatology

( $R$ L van Wanrooij MD, DSUmans, Prof J E van Hooft MD, Prof P Fockens MD) and Department of Surgery (S M van Dijk,

Prof M G Besselink MD), Amsterdam Gastroenterology Endocrinology Metabolism, Amsterdam UMC, University of Amsterdam, Amsterdam, Netherlands; Department of Gastroenterology and Hepatology, Canisius Wilhelmina Hospital, Nijmegen, Netherlands (A C IT L Tan MD); Department of Gastroenterology and Hepatology, Amphia Hospital Breda, Netherlands (TCJ Seerden MD); Department of Gastroenterology and Hepatology, Zuyderland 


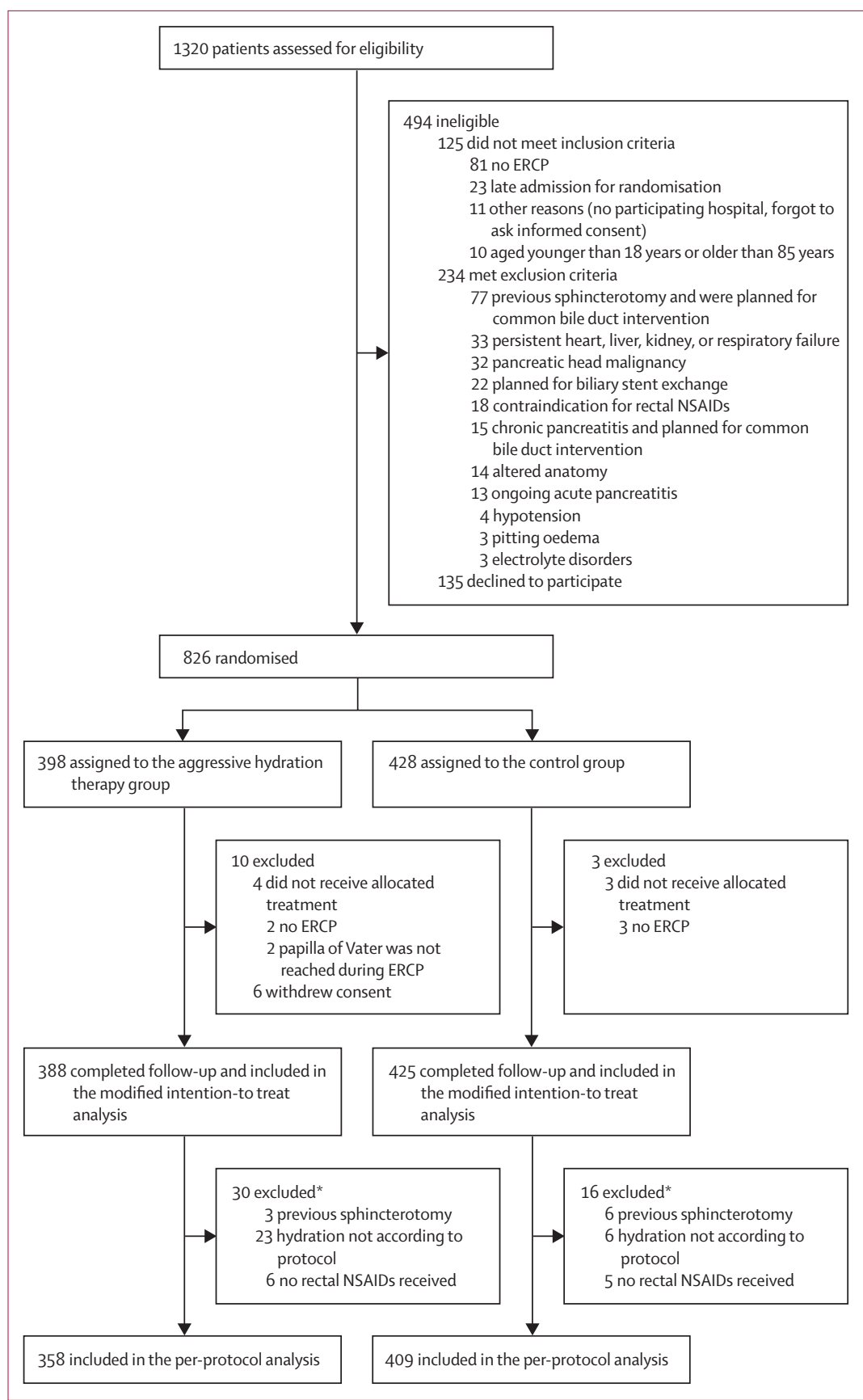

Figure 1: Trial profile

$E R C P=$ endoscopic retrograde cholangiopancreatography. NSAIDs=non-steroidal anti-inflammatory drugs.

*Some patients had more than one reason to be excluded from the per-protocol analysis.

Hospital, Heerlen, Netherlands (YCA Keulemans MD); Department of Gastroenterology and Hepatology, Netherlands Cancer Institute, Antoni van Leeuwenhoek Hospital, Amsterdam, Netherlands (T R de Wijkerslooth MD); cardioprotective aspirin]). Full eligibility criteria are listed in the appendix ( $\mathrm{p} 3$ ).

Because medicinal products used in this study (ie, Ringer's lactate solution) were under investigation, the Central Committee on Research Involving Human Subjects carried out an extra review of the trial protocol. The boards of all participating centres gave additional permission for carrying out the study in the respective centres. This investigator-initiated study was done in accordance with the principles of the Declaration of Helsinki and Dutch law regarding research involving humans. The Medical Ethical Committee United in Nieuwegein, the Netherlands, approved the trial protocol (reference number NL52341.100.15). Written informed consent was obtained from all participants. The protocol is available online.

\section{Randomisation and masking}

Patients were randomly assigned (1:1) to either aggressive periprocedural hydration combined with rectal NSAIDs (aggressive hydration group) or rectal NSAIDs alone (control group). Patients were randomly assigned centrally by the study coordinator using a web-based computer program with concealed, permuted blocks of varying sizes (two, four, or six). Randomisation was stratified according to treatment centre. Masking of treating staff and patients was deemed impracticable because visible volume input and urine volume output greatly differed between groups. However, a masked adjudication committee evaluated all primary and secondary outcomes. Furthermore, all statisticians were masked for the prophylaxis given.

\section{Procedures}

All ERCPs were carried out by or under the direct supervision of an experienced interventional endoscopist, defined as having a lifetime exposure of more than 400 ERCPs and having done more than 50 ERCPs yearly for the past 3 years. The aggressive hydration group received $100 \mathrm{mg}$ of rectal NSAIDs (diclofenac or indomethacin $)^{29}$ within 30 min before or after procedure, combined with periprocedural hydration with intravenous $20 \mathrm{~mL} / \mathrm{kg}$ Ringer's lactate solution within $60 \mathrm{~min}$ from the start of ERCP (endoscope-mouth contact) directly followed by $3 \mathrm{~mL} / \mathrm{kg}$ per h for $8 \mathrm{~h}$. The control group received $100 \mathrm{mg}$ of rectal NSAIDs alone (within $30 \mathrm{~min}$ before or after procedure) with a restricted intravenous fluid infusion with normal saline (maximum of $1.5 \mathrm{~mL} / \mathrm{kg}$ per h or $3 \mathrm{~L}$ per $24 \mathrm{~h}$ ). ${ }^{1,2,27}$ Decisions regarding placement of pancreatic duct stents were at the discretion of the attending endoscopist. After ERCP, all patients were admitted to hospital, regardless of symptoms, for $24 \mathrm{~h}$ to evaluate the two criteria that constituted the primary endpoint by measuring the concentration of amylase, lipase, or both in blood and to assess the appearance of upper abdominal pain. Thereafter, or if patients developed complications beforehand, intravenous fluid administration was at the discretion of the treating clinician. A second night of hospital treatment was indicated when a patient met the two criteria of post-ERCP pancreatitis. A prolonged hospital stay due to other indications was at the discretion of the treating clinician. Patients were followed up for 180 days after randomisation and were phoned 30,90 , and 180 days after the ERCP procedure. 
All data were collected prospectively using standardised digital case record forms and were verified by the study coordinator through patient chart review of all hospital contacts between randomisation and the end of follow-up. Quality of life was assessed with two generic questionnaires (EQ-5D and the Medical Outcome Study 36-Item Short Form) and the Standardised Instrument for Measuring and Valuing Health-Related Productivity Losses at 1, 3, and 6 months after randomisation.

\section{Outcomes}

The primary endpoint was post-ERCP pancreatitis according to the Cotton criteria-ie, the presence of new onset of upper abdominal pain suggestive of pancreatitis requiring extension of hospital stay for at least 2 nights; and elevation of pancreatic enzymes (amylase, lipase, or both) to more than three times the institutional upper limit of normal $24 \mathrm{~h}$ after ERCP. ${ }^{30}$ Secondary endpoints were severity of post-ERCP pancreatitis according to the Cotton criteria ${ }^{30}$ and revised Atlanta criteria, ${ }^{31,32}$ ERCPrelated complications according the Cotton criteria, ${ }^{30}$ hydration-related complications, pancreatic insufficiency, and duration of hospital stay (appendix pp 4-7). A masked adjudication committee consisting of five endoscopists, a radiologist, and a nephrologist individually evaluated all primary and secondary outcomes. Disagreements were resolved during a consensus meeting.

Serious adverse events were defined as events that were fatal or life threatening, that resulted in clinically significant or persistent disability, that required hospital admission or a prolonged hospital stay, or that were judged by the investigator to represent a clinically significant hazard or harm to the patient that might require medical or surgical intervention. Serious adverse events were reported by treating clinicians to the study coordinator and verified by patient chart review. All events were reported to the Dutch Central Committee for Research Involving Human Subjects.

\section{Statistical analysis}

We believe that aggressive periprocedural hydration would be a useful addition to rectal NSAIDs if it has a similar relative risk reduction. ${ }^{33}$ We assumed that a relative risk reduction of $60 \%$ would be realistic, based on the assumption that rectal NSAIDs in combination with aggressive periprocedural hydration would yield a similar relative risk reduction to rectal NSAIDs compared with placebo in previous studies. ${ }^{19,34,35}$ This minimal clinically important difference would cause the incidence of postERCP pancreatitis to decrease from $8.0 \%$ in the control group to $3 \cdot 2 \%$ in the aggressive hydration group, with a $4.8 \%$ absolute risk reduction. We calculated a sample size of 720 patients would be needed to detect a $60 \%$ relative reduction in post-ERCP pancreatitis in the aggressive hydration group (from $8 \%$ to $3 \cdot 2 \%$ ), with a power of $80 \%$ and a two-sided $\alpha$ level of $0 \cdot 05$. To allow for unexpected dropout and missing data, we increased the target sample size by $15 \%$ resulting in a final number of 826 patients (413 per group).

Patient recruitment and the association of serious adverse events with the intervention were overseen by an independent data safety monitoring board (DSMB). All events were reported to the DSMB (who were not masked) after the inclusion of 50,150, 413, and 650 patients. An independent statistician did an interim analysis after 413 patients were included, which allowed us to include
Department of Gastroenterology and Hepatology, Albert Schweitzer Hospital, Dordrecht, Netherlands (W van de Vrie MD); Department of Anaesthesiology (N D L Hallensleben) and Department of Gastroenterology and Hepatology

\begin{tabular}{|c|c|c|}
\hline & $\begin{array}{l}\text { Aggressive hydration } \\
\text { group }(n=388)\end{array}$ & $\begin{array}{l}\text { Control group } \\
(n=425)\end{array}$ \\
\hline Age, years & $57(44-71)$ & $60(49-71)$ \\
\hline \multicolumn{3}{|l|}{ Sex } \\
\hline Female & $232(60 \%)$ & $250(59 \%)$ \\
\hline Male & $156(40 \%)$ & $175(41 \%)$ \\
\hline Body-mass index, $\mathrm{kg} / \mathrm{m}^{2 *}$ & $26.7(24-30 \cdot 4)$ & $26 \cdot 9(23 \cdot 8-30 \cdot 3)$ \\
\hline Previous cholecystectomy & $112(29 \%)$ & $116(27 \%)$ \\
\hline \multicolumn{3}{|l|}{ ASA class on admission } \\
\hline I: healthy status & $90(23 \%)$ & $103(24 \%)$ \\
\hline II: mild systemic disease & $235(61 \%)$ & $255(60 \%)$ \\
\hline III: severe systemic disease & $63(16 \%)$ & $67(16 \%)$ \\
\hline $\begin{array}{l}\text { IV: severe systemic disease that is constant } \\
\text { threat to life }\end{array}$ & 0 & 0 \\
\hline \multicolumn{3}{|l|}{ Smoker } \\
\hline Current & $76 / 351(22 \%)$ & $95 / 378(25 \%)$ \\
\hline Past & $79 / 351(23 \%)$ & $84 / 378(22 \%)$ \\
\hline Never & $196 / 351(56 \%)$ & $199 / 378(53 \%)$ \\
\hline Alcohol abuse $\dagger$ & $59 / 353(17 \%)$ & $67 / 369(18 \%)$ \\
\hline \multicolumn{3}{|l|}{ ERCP indication } \\
\hline Common bile duct stones (or suspicion of) & $307(79 \%)$ & $342(80 \%)$ \\
\hline Cholangitis & $45(12 \%)$ & $46(11 \%)$ \\
\hline Metastatic cancer & $6(2 \%)$ & $5(1 \%)$ \\
\hline Cholangiocarcinoma & $7(2 \%)$ & $7(2 \%)$ \\
\hline Postoperative bile leak & $4(1 \%)$ & $8(2 \%)$ \\
\hline Sphincter of Oddi dysfunction (or suspicion of) & $5(1 \%)$ & $5(1 \%)$ \\
\hline Other & $14(4 \%)$ & $12(3 \%)$ \\
\hline \multicolumn{3}{|l|}{ Complexity of ERCP 39} \\
\hline 1 & $20(5 \%)$ & $31(7 \%)$ \\
\hline 2 & $337(87 \%)$ & $353(83 \%)$ \\
\hline 3 & $30(8 \%)$ & $39(9 \%)$ \\
\hline 4 & $1(<1 \%)$ & $2(<1 \%)$ \\
\hline Common bile duct cannulation achieved & $357(92 \%)$ & $395(93 \%)$ \\
\hline Pancreatic duct stent placement & $23(6 \%)$ & $26(6 \%)$ \\
\hline Pancreatic duct cannulation (unintentional) & $157(40 \%)$ & $158(37 \%)$ \\
\hline Pancreatic duct contrast injections (unintentional) & $58(15 \%)$ & $73(17 \%)$ \\
\hline Difficult cannulation $\ddagger$ & $113 / 378(30 \%)$ & $123 / 417(29 \%)$ \\
\hline Sphincter of Oddi dysfunction & $3(1 \%)$ & $5(1 \%)$ \\
\hline \multicolumn{3}{|c|}{$\begin{array}{l}\text { Data are median (IQR) or } \mathrm{n}(\%) \text {. Percentages might not sum to } 100 \% \text { because of rounding. ASA=American Society of } \\
\text { Anaesthesiologists. ERCP=endoscopic retrograde cholangiopancreatography. }{ }^{*} \text { Assessed in } 387 \text { patients in the } \\
\text { aggressive hydration group and } 417 \text { patients in the control group. †According to the US National Institute on Alcohol } \\
\text { Abuse and Alcoholism (more than three drinks on any single day and more than seven drinks per week for women; } \\
\text { more than four drinks on any single day and more than } 14 \text { drinks per week for men). } \neq \text { Difficult cannulation was defined } \\
\text { as more than five attempts. }\end{array}$} \\
\hline
\end{tabular}


(Prof M J Bruno MD), Erasmus Medical Centre, Rotterdam, Netherlands; Institute for Environmental Studies, Vrije Universiteit, Amsterdam, Netherlands

(R L Sperna Weiland MSc); Department of

Gastroenterology and Hepatology, Leiden University Medical Centre, Leiden, Netherlands

(ProfJ E van Hooft); Department of Surgery, University Medical Centre Utrecht, Utrecht, Netherlands

(Prof H C van Santvoort)

Correspondence to:

Dr Erwin J M van Geenen, Department of Gastroenterology and Hepatology, Radboud University Medical Center, 6500 HB Nijmegen, Netherlands erwin.vangeenen@ radboudumc.nl

See Online for appendix For the protocol see https://trialsjournal. biomedcentral.com/ articles/10.1186/ s13063-018-2583-x an option to stop the trial early for efficacy. ${ }^{36}$ We used a Peto approach to test for a beneficial effect (symmetrical stopping boundaries at $\mathrm{p}<0 \cdot 001$ ); there was no assessment of futility. ${ }^{37,38}$ The access to the result of the interim analysis was limited to the study coordinator and the DSMB to minimise potential operational bias.

Baseline variables are expressed as mean with SD or median with IQR. All primary analyses were done in the modified intention-to-treat population and the perprotocol analysis population. The modified intention-totreat analysis was based on randomly assigned patients who underwent an ERCP and for whom data regarding the primary outcome were available. For example, we excluded patients in whom the duodenum was not reached and the papilla of Vater was not manipulated during ERCP, as they cannot develop post-ERCP pancreatitis. In the modified intention-to-treat analysis, data were analysed according to the treatment to which the patient was assigned by randomisation. In the perprotocol analysis, we excluded patients who had a previous sphincterotomy, patients who did not receive rectal NSAIDs, or in case hydration was not given according to protocol. The primary endpoint was analysed using Fisher's exact test. The secondary endpoints were compared between treatment groups by the Student's $t$ test, Wilcoxon rank-sum test, Pearson's $\chi^{2}$ test, or Fisher's exact test, as appropriate. The amount of fluid given in the first $24 \mathrm{~h}$ from the start of ERCP were monitored for both groups. We compared the differences using Wilcoxon rank-sum test. Results are presented as relative risks with corresponding $95 \%$ CIs. We made no adjustments for multiple testing and did not correct for any potential bias introduced by our interim analysis because we considered the bias to be negligible. All analyses were done by an independent statistician.
We did a predefined subgroup analyses for age, sex, and pancreatic duct stent placement. Additionally, we did post-hoc analyses on risk factors for post-ERCP pancreatitis: a history of pancreatitis, difficult cannulation of the common bile duct, cannulation of the pancreatic duct, pancreatic contrast injection, and trainee involvement. ${ }^{7}$ All subgroup analyses were evaluated for confounding and whether an interaction effect was present with aggressive periprocedural hydration in combination with rectal NSAIDs by testing for significance of a corresponding interaction term following a log binominal regression model. All statistical analyses were done with $\mathrm{R}$ (version 3.6.2), with significance set at a two-sided $\alpha$ level of $5 \%$.

This trial is registered with the ISRCTN registry, number ISRCTN13659155.

\section{Role of the funding source}

The funders of the study had no role in study design, data collection data analysis, data interpretation, or writing of the report.

\section{Results}

Between June 5, 2015, and June 6, 2019, 1320 patients were assessed for eligibility, of whom 826 underwent randomisation (figure 1; appendix p 8). 13 patients were excluded from our intention-to-treat population: six patients withdrew informed consent before ERCP and seven patients did not undergo ERCP and therefore were unable to be assessed for the primary endpoint (appendix pp 9-10). 388 patients received aggressive periprocedural hydration and 425 patients served as controls in the modified intention-to-treat analysis Patients had a median age of 59 years (IQR 46-71) and 482 (59\%) were women. Baseline characteristics did not

\begin{tabular}{|c|c|c|c|c|}
\hline & $\begin{array}{l}\text { Aggressive hydration } \\
\text { group }(n=388)\end{array}$ & $\begin{array}{l}\text { Control group } \\
(n=425)\end{array}$ & Relative risk $(95 \% \mathrm{Cl})$ & p value* \\
\hline Primary outcome: post-ERCP pancreatitis & $30(8 \%)$ & $39(9 \%)$ & $0.84(0.53-1 \cdot 33)$ & 0.53 \\
\hline Delayed ( $>48 \mathrm{~h}$ after ERCP) post-ERCP pancreatitis & $1(<1 \%)$ & $1(<1 \%)$ & .. & .. \\
\hline \multicolumn{5}{|l|}{ Secondary outcomes } \\
\hline Post-ERCP pancreatitis severity according to Cotton criteria $\mathrm{a}^{30}$ &.. & .. & .. & .. \\
\hline Mild & $9(2 \%)$ & $7(2 \%)$ & $1.38(0.52-3 \cdot 66)$ & 0.52 \\
\hline Moderate or severe & $21(5 \%)$ & $32(8 \%)$ & $0.72(0.42-1.23)$ & 0.23 \\
\hline $\begin{array}{l}\text { Post-ERCP pancreatitis severity according to revised Atlanta } \\
\text { criteria }^{31,32}\end{array}$ &.. & .. & .. & .. \\
\hline Mild & $27(7 \%)$ & $29(7 \%)$ & $1.00(0.61-1.66)$ & 0.99 \\
\hline Moderate or severe & $3(1 \%)$ & $10(2 \%)$ & $0.33(0.09-1.19)$ & 0.089 \\
\hline Exocrine insufficiency $\ddagger$ & 0 & $2(<1 \%)$ & $0.40(0.02-7.69)$ & 0.25 \\
\hline Endocrine insufficiency $₫$ & $4(1 \%)$ & $3(1 \%)$ & $1.70(0.44-6.66)$ & 0.49 \\
\hline Duration of hospital stay, nights & $1(1-2)$ & $1(1-2)$ & .. & $0.77 \|$ \\
\hline \multicolumn{5}{|c|}{$\begin{array}{l}\text { Data are } \mathrm{n}(\%) \text { or median (IQR) unless otherwise stated. Percentages might not sum to } 100 \% \text { because of rounding. * } \mathrm{p} \text { values were based on Fisher's exact test. } \dagger \text { Defined as a } \\
\text { faecal pancreatic elastase- } 1 \text { concentration of less than } 200 \mu \mathrm{g} / \mathrm{g} 180 \text { days after diagnosis of pancreatitis. } \neq \text { Assessed in } 11 \text { patients in the aggressive hydration group and in } \\
21 \text { patients in the control group. SDefined as a glycated haemoglobin concentration of more than } 42 \mathrm{mmol} / \mathrm{mol}(6 \%) 180 \text { days after diagnosis of pancreatitis. } \uparrow \text { Assessed in } \\
14 \text { patients in the aggressive hydration group and in } 20 \text { patients in the control group. \|lp value is based on Wilcoxon rank-sum test. }\end{array}$} \\
\hline
\end{tabular}


differ between groups (table 1; appendix p 11). Diclofenac was the only rectal NSAID to be administered.

All ERCP characteristics were similar between the groups (appendix p 12). Suspicion of common bile duct stones was the predominant indication for ERCP (table 1). The use of pancreatic duct stents was limited and similar between both groups (table 1). The aggressive hydration group received a higher fluid volume in the first $24 \mathrm{~h}$ from the start of ERCP than the control group (3562 mL [IQR 3124-4101] vs $400 \mathrm{~mL}$ [0-1640], $\mathrm{p}<0 \cdot 0001$; appendix $\mathrm{p} 15)$.

A protocol violation occurred for $46(6 \%)$ of 813 patients: nine patients had a previous sphincterotomy, 11 patients did not receive rectal NSAIDs, and hydration was not given according to the protocol in 29 patients (appendix p 10).

There were no reasons for terminating the trial after the interim analysis: post-ERCP pancreatitis occurred in $23(11 \%)$ of the 212 patients in the control group, as compared with $16(8 \%)$ of the 201 patients in the aggressive hydration group $(\mathrm{p}=0 \cdot 40)$.

Post-ERCP pancreatitis occurred in $30(8 \%)$ patients in the aggressive hydration group, as compared with 39 (9\%) patients in the control group (relative risk [RR] 0.84, 95\% CI 0.53-1.33; $\mathrm{p}=0.53$; table 2 ). Two pancreatitis events had a late onset ( $>48 \mathrm{~h}$ after the ERCP procedure), one in each group. Moderate or severe post-ERCP pancreatitis based on Cotton criteria ${ }^{30}$ was seen in 53 patients: $21(5 \%)$ in the aggressive hydration group versus $32(7 \%)$ in the control group (RR $0 \cdot 72,95 \% \mathrm{CI}$ $0 \cdot 42-1 \cdot 23 ; \mathrm{p}=0 \cdot 23)$. Moderate or severe post-ERCP pancreatitis based on revised Atlanta criteria ${ }^{31,32}$ was seen in 13 patients: three $(1 \%)$ in the aggressive hydration group versus ten (2\%) in the control group (RR 0.33 , 95\% CI $0 \cdot 09-1 \cdot 19 ; p=0 \cdot 089)$. The median duration of hospital stay was 1 night (IQR 1-2; $\mathrm{p}=0 \cdot 77$ ) for both groups. Results did not change in the per-protocol analysis (appendix p 13).

No significant differences were observed between the groups with regard to hydration-related complications (RR 0.99, 95\% CI 0.59-1.64; $\mathrm{p}=1 \cdot 00)$, ERCP-related complications $(0 \cdot 90,0 \cdot 62-1 \cdot 31 ; p=0 \cdot 62)$, exocrine insufficiency $(0.40,0 \cdot 02-7 \cdot 69 ; \mathrm{p}=0 \cdot 25)$, endocrine insufficiency $(1 \cdot 70,0.44-6 \cdot 66 ; \mathrm{p}=0.49)$, intensive care unit admission $(0 \cdot 37,0 \cdot 07-1 \cdot 80 ; \mathrm{p}=0 \cdot 22)$, or 30 -day mortality $(0 \cdot 95,0 \cdot 50-1 \cdot 83 ; \mathrm{p}=1 \cdot 00$; table 3 ; appendix pp 16-18). None of the deaths was related to aggressive hydration (appendix p 14).

In the predefined subgroup analyses for age, sex, and pancreatic duct stent placement, we observed no significant interaction term. Furthermore, no significant interaction terms were observed with respect to a history of pancreatitis, difficult cannulation of the common bile duct, cannulation of the pancreatic duct, pancreatic contrast injection, and trainee involvement (figure 2).

\section{Discussion}

This multicentre randomised study shows that the combination of aggressive periprocedural hydration with

\begin{tabular}{|c|c|c|c|c|}
\hline & $\begin{array}{l}\text { Aggressive } \\
\text { hydration group } \\
(\mathrm{n}=388)\end{array}$ & $\begin{array}{l}\text { Control group } \\
(n=425)\end{array}$ & $\begin{array}{l}\text { Relative risk } \\
(95 \% \mathrm{Cl})\end{array}$ & p valuet \\
\hline ERCP-related complications & $16(4 \%)$ & $21(5 \%)$ & $0.90(0.62-1.31)$ & 0.62 \\
\hline Cholangitis & $2(1 \%)$ & $6(1 \%)$ & .. & 0.44 \\
\hline Bleeding & $10(3 \%)$ & $12(3 \%)$ &.. & 0.53 \\
\hline Perforation & $5(1 \%)$ & $4(1 \%)$ & .. & 0.49 \\
\hline Hydration-related complications & $8(2 \%)$ & $9(2 \%)$ & $0.99(0.59-1.64)$ & 1.00 \\
\hline Pulmonary oedema & $3(1 \%)$ & $5(1 \%)$ & & 0.80 \\
\hline Peripheral oedema & $6(2 \%)$ & $3(1 \%)$ & & 0.22 \\
\hline Cardiac insufficiency & 0 & $3(1 \%)$ & .. & 0.25 \\
\hline Hypernatraemia & $1(<1 \%)$ & $1(<1 \%)$ &.. & 1.00 \\
\hline ICU admission after ERCP & $2(1 \%)$ & $6(1 \%)$ & $0.37(0.07-1.80)$ & 0.22 \\
\hline 30-day mortality & $5(1 \%)$ & $6(1 \%)$ & $0.95(0.50-1.83)$ & 1.00 \\
\hline Mortality during 180-day follow-up & $11(3 \%)$ & $12(3 \%)$ & $1.00(0.45-2.25)$ & 1.00 \\
\hline $\begin{array}{l}\text { Cholangitis during 180-day } \\
\text { follow-up }\end{array}$ & $5(1 \%)$ & $8(2 \%)$ & $0.68(0.23-2.08)$ & 0.50 \\
\hline \multicolumn{5}{|c|}{$\begin{array}{l}\text { Data are } n(\%) \text { or median (IQR). ERCP=endoscopic retrograde cholangiopancreatography. ICU=intensive care unit. } \\
\text { *This table presents the most clinically relevant serious adverse events, intervention-related events, and events that } \\
\text { appear in more than } 1 \% \text { in the study population. A complete overview of serious adverse events can be found in the } \\
\text { appendix (pp 16-18). } † \text { p values were based on Fisher's exact test. }\end{array}$} \\
\hline
\end{tabular}

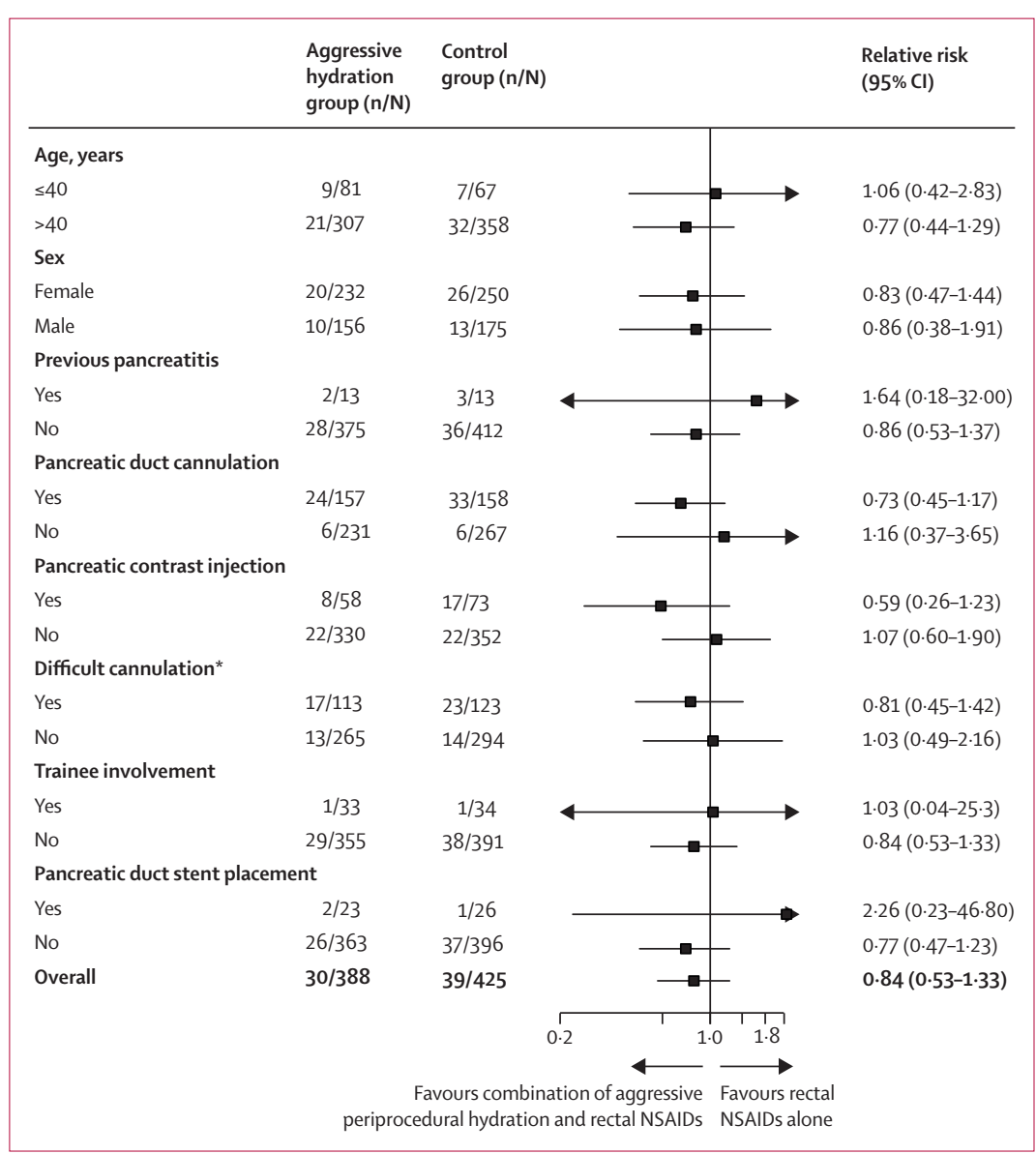

Figure 2: Relative risk of post-ERCP pancreatitis in subgroups

$E R C P=$ endoscopic retrograde cholangiopancreatography. NSAIDs=non-steroidal anti-inflammatory drugs. *Difficult cannulation was defined as more than five attempts. 
Ringer's lactate solution and rectal NSAIDs was not superior to rectal NSAIDs alone in reducing the incidence of post-ERCP pancreatitis in patients at moderate to high risk of developing this complication. Furthermore, combination therapy did not reduce the severity of postERCP pancreatitis.

ERCP is an indispensable tool to diagnose and treat pancreatobiliary tract disorders, but its inherent high risk of complications-in particular, post-ERCP pancreatitisis concerning. The identification of patient-related and procedure-related risk factors have aided in the development of risk stratification strategies to curtail complications. Despite these measures, the incidence of post-ERCP pancreatitis remains substantial. ${ }^{7}$ Rectal NSAIDs reduce the relative risk of developing post-ERCP pancreatitis by $60 \% .^{5,40}$ The prevention of severe postERCP pancreatitis remains an unmet need, which is emphasised by the substantial pancreatitis incidence of $9 \%$ in our trial despite the use of rectal NSAIDs.

Aggressive periprocedural hydration has been suggested as a non-pharmacological measure to reduce the incidence of post-ERCP pancreatitis. ${ }^{13,41}$ Aggressive hydration with Ringer's lactate solution was shown to reduce the incidence of post-ERCP pancreatitis as compared with standard hydration in patients who did not receive rectal NSAIDs in a meta-analysis (33 [6\%] of 533 vs 65 [13\%] of 514 patients; odds ratio $0.47,95 \% \mathrm{CI}$ $0 \cdot 30-0 \cdot 72 ; \mathrm{p}=0 \cdot 0006)^{13}$ and a randomised study (four [3\%] of 132 vs 15 [12\%] of 129 patients; RR $0 \cdot 26$, 95\% CI $0 \cdot 08-0 \cdot 76 ; \mathrm{p}=0 \cdot 008) .{ }^{42}$ One large randomised study showed a reduction in the incidence of moderate to severe post-ERCP pancreatitis in patients receiving aggressive periprocedural hydration compared with standard periprocedural hydration (one [0.4\%] of 255 vs five [2.0\%] of 255 patients; $\mathrm{p}=0 \cdot 04) .{ }^{43}$ These data led to international treatment guidelines to recommend aggressive periprocedural hydration to decrease the risk of post-ERCP pancreatitis. ${ }^{7,8}$

The effect of aggressive periprocedural hydration combined with rectal NSAIDs, as compared with rectal NSAIDs alone, was investigated in two smaller singlecentre randomised studies with conflicting results..$^{25,26}$ In the first study, a four-armed trial that compared aggressive normal saline hydration ( $1 \mathrm{~L}$ in $2 \mathrm{~h}$ before ERCP and $2 \mathrm{~L}$ in $16 \mathrm{~h}$ thereafter) combined with rectal NSAIDs $(n=101)$ and rectal NSAIDs alone $(n=100)$, combination therapy resulted in a reduction of pancreatitis incidence from $11 \%$ to $0 \%(\mathrm{p}=0 \cdot 001) .{ }^{25}$ In the second, two-arm trial, the authors found no difference in the occurrence of post-ERCP pancreatitis with aggressive periprocedural hydration with Ringer's lactate solution (a scheme similar to that used in this study) combined with rectal NSAIDs compared with rectal NSAIDs alone (one [0.9\%] of 107 patients $v$ s three [2.7\%] of 112 patients; $\mathrm{p}=0 \cdot 62) .{ }^{26}$ Because both trials were done in an average-risk population with a low baseline risk of pancreatitis and small sample sizes, there was a substantial chance of false-positive and false-negative findings. Both trials were single-centre studies, which limits the generalisability of their results.

The current study has some limitations. First, treating staff and patients were not masked because there were major practical issues that precluded proper masking of the aggressive hydration group, such as differences in infusion volume and urine output. To address these issues, the primary outcome included objective criteria (ie, elevation of pancreatic enzymes) and was assessed by a masked adjudication committee. Second, concurrent use of rectal NSAIDs and pancreatic duct stenting is under discussion and merits further investigation. Therefore, we left pancreatic duct stent placement to the discretion of the treating endoscopist. However, few stents were used and stent use was evenly distributed between the groups. ${ }^{44}$ Therefore, it is unlikely that pancreatic duct stent placement affected the outcomes of this trial. Third, there is no universal risk stratification system for post-ERCP pancreatitis. This potentially complicates our patient selection and sample size generation. However, the risk profile of our study population is similar to the populations of the studies that were the basis of our sample size calculation: patients with moderate to high risk who receive rectal NSAIDs. ${ }^{34,35}$ The meta-analyses mention a post-ERCP pancreatitis incidence of $8.0 \%$ and $5.7 \%$, which is similar to that in the control group (rectal NSAIDs alone) of our study ( $9 \%)$. These data are in line with those from a recent metaanalysis (mean $6 \cdot 5 \%$, range $3 \cdot 2-10 \cdot 1$ ). ${ }^{40}$

The strengths of this study include its multicentre design and the fact that our treatment design follows international recommendations on the prevention of post-ERCP pancreatitis, which increases generalisability of the results. Inclusion of patients at moderate to high risk of post-ERCP pancreatitis led to a high pancreatitis incidence and a smaller chance of a type II error. Despite the complexity of the study and various hospital settings, the hydration protocol was closely followed in 784 (96\%) of the 813 patients.

In conclusion, the combination of rectal NSAIDs and aggressive periprocedural hydration does not notably reduce the incidence of post-ERCP pancreatitis, as compared with rectal NSAIDs alone in patients with moderate to high risk of post-ERCP pancreatitis. Therefore, the burden of laborious and time-consuming hydration is not justified in patients already receiving rectal NSAIDs.

\section{Contributors}

EJMvG supervised the study. CJSW and XJNMS coordinated the trial during inclusion. RSW did the statistical analysis. CJSW checked the statistical analysis. CSW drafted the manuscript. XJNMS, EJMvG, JPHD MJB, MGB, PF, JEvH, RCV, WK, and HCTvS co-authored the manuscript. All authors critically assessed the study design, included patients in the study, edited the manuscript, and read and approved the final manuscript. CJSW, XJNMS, and EJMvG accessed and verified the data. All authors vouch for the accuracy and completeness of the data and its analyses. The corresponding author has full access to all the data in the study and had final responsibility for the decision to submit for publication. 


\section{Declaration of interests}

EJMvG reports grants from Mylan and Olympus and personal fees from MTW-Endoskopie, outside the submitted work. JPHD reports grants from Gilead, outside the submitted work. PF reports personal fees from Olympus, Cook Medical, and Ethicon Endosurgery, outside the submitted work. MJB reports personal fees from Boston Scientific, grants and personal fees from Cook Medical, and grants from Pentax Medical, 3M, InterScope, and Mylan, outside the submitted work. JEvH reports grants and personal fees from Cook Medical and personal fees from Boston Scientific and Medtronics, outside the submitted work. All other authors declare no competing interests.

\section{Data sharing}

Requests for data can be made to the corresponding author and will be discussed during a meeting of the Dutch Pancreatitis Study Group. Individual participant data that underlie the results reported in this Article, after de-identification, will be shared after approval by the Dutch Pancreatitis Study Group. Related documents, such as trial protocol and statistical analysis plan, will be available online immediately following publication without an end date to anyone who wishes to access the data.

\section{Acknowledgments}

The study was funded by the Netherlands Organisation for Health Research and Development (grant number 837001506) and the Radboud University Medical Center. We thank members of the data safety monitoring board (Ruud Loffeld [chair; Department of Gastroenterology and Hepatology, Zaans Medical Centre, Zaandam, Netherlands], Tom Nijenhuis [Department of Nephrology, Radboud University Medical Center, Nijmegen, Netherlands], David M Burger [Department of Pharmacy, Radboud University Medical Center, Nijmegen, Netherlands], and Michiel Vaneker [Department of Anesthesiology, Radboud University Medical Center, Nijmegen, Netherlands]) and the independent statistician for the interim analysis (Joanna IntHout at the Department for Health Evidence, Radboud University Medical Center, Nijmegen, Netherlands) for their services.

\section{References}

1 Peery AF, Crockett SD, Murphy CC, et al. Diseases in the United States: update 2018. Gastroenterology 2019; 156: 254-72.

2 Kochar B, Akshintala VS, Afghani E, et al. Incidence, severity, and mortality of post-ERCP pancreatitis: a systematic review by using randomized, controlled trials. Gastrointest Endosc 2015; 81: 143-49.

3 Andriulli A, Loperfido S, Napolitano G, et al. Incidence rates of post-ERCP complications: a systematic survey of prospective studies. Am J Gastroenterol 2007; 102: 1781-88.

4 Elmunzer BJ, Scheiman JM, Lehman GA, et al. A randomized trial of rectal indomethacin to prevent post-ERCP pancreatitis. N Engl J Med 2012; 366: 1414-22.

5 Serrano JPR, de Moura DTH, Bernardo WM, et al. Nonsteroidal anti-inflammatory drugs versus placebo for post-endoscopic retrograde cholangiopancreatography pancreatitis: a systematic review and meta-analysis. Endosc Int Open 2019; 7: E477-86.

6 Sugimoto M, Takagi T, Suzuki R, et al. Pancreatic stents to prevent post-endoscopic retrograde cholangiopancreatography pancreatitis: a meta-analysis. World J Metaanal 2019; 7: 249-58.

7 Chandrasekhara V, Khashab MA, Muthusamy VR, et al. Adverse events associated with ERCP. Gastrointest Endosc 2017; 85: 32-47.

8 Dumonceau J-M, Kapral C, Aabakken L, et al. ERCP-related adverse events: European Society of Gastrointestinal Endoscopy (ESGE) Guideline. Endoscopy 2020; 52: 127-49.

9 Yokoe M, Takada T, Mayumi T, et al. Japanese guidelines for the management of acute pancreatitis: Japanese Guidelines 2015. J Hepatobiliary Pancreat Sci 2015; 22: 405-32.

10 Elmunzer BJ, Serrano J, Chak A, et al. Rectal indomethacin alone versus indomethacin and prophylactic pancreatic stent placement for preventing pancreatitis after ERCP: study protocol for a randomized controlled trial. Trials 2016; 17: 120.

11 Choksi NS, Fogel EL, Cote GA, et al. The risk of post-ERCP pancreatitis and the protective effect of rectal indomethacin in cases of attempted but unsuccessful prophylactic pancreatic stent placement. Gastrointest Endosc 2015; 81: 150-55.
12 Elmunzer BJ, Higgins PDR, Saini SD, et al. Does rectal indomethacin eliminate the need for prophylactic pancreatic stent placement in patients undergoing high-risk ERCP? Post hoc efficacy and cost-benefit analyses using prospective clinical trial data. Am J Gastroenterol 2013; 108: 410-15.

13 Zhang ZF, Duan ZJ, Wang LX, Zhao G, Deng WG. Aggressive hydration with lactated ringer solution in prevention of postendoscopic retrograde cholangiopancreatography pancreatitis: a meta-analysis of randomized controlled trials. J Clin Gastroenterol 2017; 51: e17-26.

14 Senol A, Saritas U, Demirkan H. Efficacy of intramuscular diclofenac and fluid replacement in prevention of post-ERCP pancreatitis. World J Gastroenterol 2009; 15: 3999-4004.

15 Sagi SV, Schmidt S, Fogel E, et al. Association of greater intravenous volume infusion with shorter hospitalization for patients with postERCP pancreatitis. J Gastroenterol Hepatol 2014; 29: 1316-20.

16 DiMagno MJ, Wamsteker EJ, Maratt J, et al. Do larger periprocedural fluid volumes reduce the severity of postendoscopic retrograde cholangiopancreatography pancreatitis? Pancreas 2014; 43: 642-47.

17 Shaygan-Nejad A, Masjedizadeh AR, Ghavidel A, Ghojazadeh M, Khoshbaten M. Aggressive hydration with lactated Ringer's solution as the prophylactic intervention for postendoscopic retrograde cholangiopancreatography pancreatitis: a randomized controlled double-blind clinical trial. J Res Med Sci 2015; 20: 838-43.

18 Grunwald D, Wadhwa V, Sawhney MS. Hemodynamic variation and intravenous fluids administered during ERCP and the association with post-ERCP pancreatitis. Pancreas 2016; 45: 293-97.

19 Buxbaum J, Yan A, Yeh K, Lane C, Nguyen N, Laine L. Aggressive hydration with lactated Ringer's solution reduces pancreatitis after endoscopic retrograde cholangiopancreatography. Clin Gastroenterol Hepatol 2014; 12: 303-07.

20 Avila P, Holmes I, Kouanda A, Arain M, Dai SC. Practice patterns of post-ERCP pancreatitis prophylaxis techniques in the United States: a survey of advanced endoscopists. Gastrointest Endosc 2020; 91: 568-73.

21 Cuthbertson CM, Christophi C. Disturbances of the microcirculation in acute pancreatitis. Br J Surg 2006; 93: 518-30.

22 Coté GA, Sagi SV, Schmidt SE, et al. Early measures of hemoconcentration and inflammation are predictive of prolonged hospitalization from post- endoscopic retrograde cholangiopancreatography pancreatitis. Pancreas 2013; 42: 850-54.

23 Gross V, Leser HG, Heinisch A, Schölmerich J. Inflammatory mediators and cytokines-new aspects of the pathophysiology and assessment of severity of acute pancreatitis? Hepatogastroenterology 1993; 40: 522-30.

24 Mäkelä A, Kuusi T, Schröder T. Inhibition of serum phospholipase-A2 in acute pancreatitis by pharmacological agents in vitro. Scand J Clin Lab Invest 1997; 57: 401-07.

25 Hosseini M, Shalchiantabrizi P, Yektaroudy K, Dadgarmoghaddam M, Salari M. Prophylactic effect of rectal indomethacin administration, with and without intravenous hydration, on development of endoscopic retrograde cholangiopancreatography pancreatitis episodes: a randomized clinical trial. Arch Iran Med 2016; 19: 538-43.

26 Hajalikhani M, Emami MH, Khodadoostan M, Shavakhi A, Rezaei M, Soluki R. Combination of diclofenac and aggressive hydration for the prevention of post-ERCP pancreatitis. Gastroenterol Hepatol Bed Bench 2018; 11: 319-24.

27 Smeets XJNM, da Costa DW, Fockens P, et al. Fluid hydration to prevent post-ERCP pancreatitis in average- to high-risk patients receiving prophylactic rectal NSAIDs (FLUYT trial): study protocol for a randomized controlled trial. Trials 2018; 19: 207.

28 Schneider A, Löhr JM, Singer MV. The M-ANNHEIM classification of chronic pancreatitis: introduction of a unifying classification system based on a review of previous classifications of the disease. J Gastroenterol 2007; 42: 101-19.

29 Dumonceau J-M, Andriulli A, Elmunzer BJ, et al. Prophylaxis of postERCP pancreatitis: European Society of Gastrointestinal Endoscopy (ESGE) Guideline-updated June 2014. Endoscopy 2014; 46: 799-815.

30 Cotton PB, Lehman G, Vennes J, et al. Endoscopic sphincterotomy complications and their management: an attempt at consensus. Gastrointest Endosc 1991; 37: 383-93. 
31 Banks PA, Bollen TL, Dervenis C, et al. Classification of acute pancreatitis-2012: revision of the Atlanta classification and definitions by international consensus. Gut 2013; 62: 102-11.

32 Smeets X, Bouhouch N, Buxbaum J, et al. The revised Atlanta criteria more accurately reflect severity of post-ERCP pancreatitis compared to the consensus criteria. United European Gastroenterol J 2019; 7: 557-64.

33 Coté GA. Intravenous hydration for the prevention of postendoscopic retrograde cholangiopancreatography pancreatitis. Gastroenterology 2014; 146: 581-82.

34 Ding X, Chen M, Huang S, Zhang S, Zou X. Nonsteroidal antiinflammatory drugs for prevention of post-ERCP pancreatitis: a meta-analysis. Gastrointest Endosc 2012; 76: 1152-59.

35 Akbar A, Abu Dayyeh BK, Baron TH, Wang Z, Altayar O, Murad MH. Rectal nonsteroidal anti-inflammatory drugs are superior to pancreatic duct stents in preventing pancreatitis after endoscopic retrograde cholangiopancreatography: a network metaanalysis. Clin Gastroenterol Hepatol 2013; 11: 778-83.

36 Dimairo M, Pallmann P, Wason J, et al. The Adaptive designs CONSORT Extension (ACE) statement: a checklist with explanation and elaboration guideline for reporting randomised trials that use an adaptive design. BMJ 2020; 369: m115.

37 Peto R, Pike MC, Armitage P, et al. Design and analysis of randomized clinical trials requiring prolonged observation of each patient. I. Introduction and design. Br J Cancer 1976; 34: 585-612.
38 Haybittle JL. Repeated assessment of results in clinical trials of cancer treatment. Br J Radiol 1971; 44: 793-97.

39 Cotton PB, Eisen G, Romagnuolo J, et al. Grading the complexity of endoscopic procedures: results of an ASGE working party. Gastrointest Endosc 2011; 73: 868-74.

40 Patai Á, Solymosi N, Mohácsi L, Patai ÁV. Indomethacin and diclofenac in the prevention of post-ERCP pancreatitis: a systematic review and meta-analysis of prospective controlled trials. Gastrointest Endosc 2017; 85: 1144-56.e1.35

41 Smeets XJNM, da Costa DW, Besselink MG, et al. Systematic review: periprocedural hydration in the prevention of post-ERCP pancreatitis. Aliment Pharmacol Ther 2016; 44: 541-53.

42 Park CH, Paik WH, Park ET, et al. Aggressive intravenous hydration with lactated Ringer's solution for prevention of post-ERCP pancreatitis: a prospective randomized multicenter clinical trial. Endoscopy 2018; 50: 378-85.

43 Choi JH, Kim HJ, Lee BU, Kim TH, Song IH. Vigorous periprocedural hydration with lactated Ringer's solution reduces the risk of pancreatitis after retrograde cholangiopancreatography in hospitalized patients. Clin Gastroenterol Hepatol 2017; 15: 86-92.

44 Smith ZL, Elmunzer BJ, Cooper GS, Chak A. Real-world practice patterns in the era of rectal indomethacin for prophylaxis against post-ERCP pancreatitis in a high-risk cohort. Am J Gastroenterol 2020; 115: 934-40 